\title{
An assessment of some closed-form expressions for the Voigt function
}

\author{
Franz Schreier \\ DLR - German Aerospace Center, Remote Sensing Technology Institute, \\ Oberpfaffenhofen, 82234 Weßling, Germany
}

\begin{abstract}
Using triangular function approximations of the Gaussian, closed-form analytical representations of the Voigt function $K(x, y)$ in terms of elementary functions can be derived. The performance of the approximations is studied by comparison with more exact numerical algorithms for the closely related complex error function, indicating serious problems for small $y$.
\end{abstract}

Keywords: Complex error function; Complex probability function; Plasma dispersion function; Faddeyeva function

doi: http://dx.doi.org/10.1016/j.jqsrt.2016.02.016

\section{Introduction}

The convolution integral of a Lorentzian and Gaussian function is important in many branches of physics and related fields. The integral does not have a closed-form analytical solution, and computational approaches for the Voigt function [1, 2] have been discussed in well over a hundred papers. Whereas most "state-of-the art" modern algorithms employ sophisticated numerical techniques [e.g. 3, 4, 5] to evaluate the closely related complex error function (also complex probability function or plasma dispersion function, cf. e.g., 6, 7), closed analytical approximations in terms of elementary functions have been proposed by several authors. Whiting [8, Peyre and Principi [9], Kielkopf [10], Wertheim et al. [11, Thompson et al. [12, Teodorescu et al. [13, Titov and Haus [14, Ida et al. [15] and Liu et al. [16] suggested linear combinations of Lorentzian and Gaussian functions (sometimes called "pseudo-Voigt" functions, occasionally with a correction term), Flores-Llamas et al. [17] proposed a sum of the Lorentzian and its derivatives, Melcher and Gerth [18] fitted the Voigt functions by generalized Lorentz functions of the $n$-th degree, and Martin and Puerta [19, 20, Puerta and Martin 21, 22] used superpositions of two to four Lorentzians.

\footnotetext{
${ }^{*}$ Corresponding author

Email address: franz.schreier@dlr.de (Franz Schreier)
}

Preprint submitted to J. Quant. Spectr. \& Rad. Trans. February 1, accepted February 5, 2016 


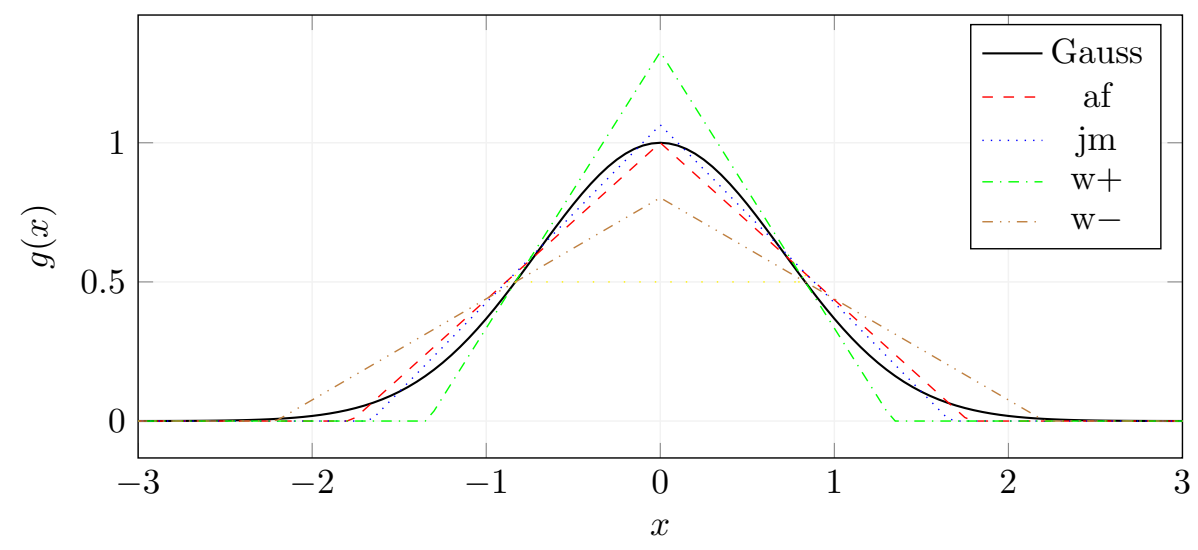

Figure 1: Comparison of triangular functions used to approximate the Gaussian.

More recently, Jiménez-Mier 23] derived an approximation for the plasma dispersion function in terms of logarithms utilizing a triangular function approximation for the Gaussian and claimed that this is "particularly good in regions where the evaluation of the plasma dispersion function is difficult." In the "Atlas of Functions" Oldham et al. 24] present a slightly different triangular approximation for the Gaussian that can also be used to approximate the Voigt function.

In this note we provide an assessment of these two approximations for the Voigt function. Additionally, we derive another approximation using a further triangular representation of the Gaussian. In the following section we briefly review the definitions, assumptions, and resulting approximations, and present comparisons with an accurately evaluated Voigt function in section 3 Our conclusions are given in the final section 4 .

\section{Theory}

The Voigt function (normalized to $\sqrt{\pi}$ ) is defined by

$$
K(x, y)=\frac{y}{\pi} \int_{-\infty}^{\infty} \frac{\mathrm{e}^{-t^{2}}}{(x-t)^{2}+y^{2}} \mathrm{~d} t
$$

where $x$ is a measure of the distance to the center peak, and $y$ is essentially the ratio of the Lorentzian and Gaussian width.

A closed-form solution of the integral can be readily obtained using a simple approximation for the Gauss function $g(x)=\exp \left(-x^{2}\right)$. Jiménez-Mier [23] suggested "a triangular function of the same width and area"

$$
g_{\mathrm{jm}}(x)= \begin{cases}\frac{1}{2} \sqrt{\frac{\pi}{\ln 2}}\left(1-\frac{|x|}{2 \sqrt{\ln 2}}\right) & |x| \leq 2 \sqrt{\ln 2} \\ 0 & \text { else. }\end{cases}
$$


In fact, this approximation drops by half at the right position $x_{1 / 2}=\sqrt{\ln 2}$ similar to the Gaussian, but because of the overestimation of the function value at the origin, the half width value is overestimated as well. Likewise, the $1 / e$ width is too high, $g_{\mathrm{jm}}(1)=0.425$ instead of $g(1)=1 / e=0.368$. On the other hand, the approximation given in the "Atlas of Functions" [24]

$$
g_{\text {af }}(x)= \begin{cases}1-|x| / \sqrt{\pi} & |x| \leq \sqrt{\pi} \\ 0 & \text { else }\end{cases}
$$

has the correct center peak value $g_{\text {af }}(0)=1$, but a wrong half width. Both approximations have the correct definite integral $\int \exp \left(-x^{2}\right) \mathrm{d} x=\int g_{\text {approx }}(x) \mathrm{d} x=$ $\sqrt{\pi}$.

A triangular approximation with the right half width $g_{\mathrm{w}}(\sqrt{\ln 2})=1 / 2$ can be easily constructed using the ansatz

$$
g_{\mathrm{w}}(x)= \begin{cases}a-b|x| & |x| \leq a / b \\ 0 & \text { else. }\end{cases}
$$

Using the integral as a second condition leads to a quadratic expression for $b$ with two solutions

$$
\begin{array}{ll}
b_{ \pm}=\frac{\sqrt{\pi}-\sqrt{\ln 2} \pm \sqrt{\pi-2 \sqrt{\pi \ln 2}}}{2 \ln 2} & =\left\{\begin{array}{l}
0.993 \\
0.363
\end{array}\right. \\
a_{ \pm}=\frac{1}{2}+\sqrt{\ln 2} b_{ \pm} & =\left\{\begin{array}{l}
1.326 \\
0.803
\end{array}\right.
\end{array}
$$

The function values at the origin, $g_{\mathrm{w}}(0)=a_{ \pm}$, strongly deviate from one, and the zeros are at the positions $a_{+} / b_{+}=1.336$ and $a_{-} / b_{-}=2.209$, i.e. there is a short \& fat and a tall \& slim triangle, see Fig. 1.

Now let $\tau$ denote the extension of the triangular function. Then evaluation of the integral in (1) gives

$$
\begin{aligned}
I(x, y, \tau)= & \int_{-\tau}^{\tau} \frac{1-|t| / \tau}{(x-t)^{2}+y^{2}} \mathrm{~d} t \\
= & \frac{\tau-x}{\tau y} \arctan \frac{\tau-x}{y}+\frac{\tau+x}{\tau y} \arctan \frac{\tau+x}{y}-\frac{2 x}{\tau y} \arctan \frac{x}{y} \\
& +\frac{1}{\tau} \ln \left(x^{2}+y^{2}\right)-\frac{1}{2 \tau}\left[\ln \left[(x+\tau)^{2}+y^{2}\right]+\ln \left[(x-\tau)^{2}+y^{2}\right]\right]
\end{aligned}
$$

resulting in the Voigt function approximations

$$
\begin{aligned}
K_{\mathrm{jm}}(x, y) & =\frac{y}{2 \sqrt{\pi \ln 2}} I(x, y, 2 \sqrt{\ln 2}), \\
K_{\mathrm{af}}(x, y) & =\frac{y}{\pi} I(x, y, \sqrt{\pi}), \\
K_{\mathrm{w} \pm}(x, y) & =\frac{a_{ \pm} y}{\pi} I\left(x, y, \frac{a_{ \pm}}{b_{ \pm}}\right) .
\end{aligned}
$$


These approximations are clearly symmetric in $x$ and should have the correct normalization $\sqrt{\pi}$ because of the normalization of the triangular functions (see next section). The peak value for $x=0$ is given by

$$
\begin{aligned}
K_{\mathrm{jm}}(0, y) & =\frac{\arctan (2 \sqrt{\ln 2} / y)}{\sqrt{\pi \ln 2}}+\frac{y}{4 \sqrt{\pi} \ln 2}\left[\ln \left(y^{2}\right)-\ln \left(4 \ln 2+y^{2}\right)\right] \\
K_{\mathrm{af}}(0, y) & =\frac{2}{\pi} \arctan \frac{\sqrt{\pi}}{y}+\frac{y}{\pi^{3 / 2}}\left[\ln y^{2}-\ln \left(\pi+y^{2}\right)\right] \\
K_{\mathrm{w}}(0, y) & =\frac{2 a}{\pi} \arctan \frac{a}{b y}+\frac{b y}{\pi}\left[\ln \left(y^{2}\right)-\ln \left(\left(\frac{a}{b}\right)^{2}+y^{2}\right)\right]
\end{aligned}
$$

in contrast to the exact value $K(0, y)=\mathrm{e}^{y^{2}} \operatorname{erfc}(y)$ (the \pm subscript has been omitted in the last equation). Since the logarithmic terms shown in the square brackets approximately cancel each other out and $\arctan (x)=\pi / 2-1 / x$ for $x \rightarrow \infty$ all approximations tend to zero for very large $x$.

\section{Results}

The range of $y$ values encountered in atmospheric spectroscopy and related areas spans many orders of magnitude [25. Letchworth and Benner [26] found $y$ values as small as $2 \cdot 10^{-8}$, and Wells [27] stated that the range of $10^{-4}<y<$ 125 for Earth "atmospheric radiance transmittance calculations ... was much greater than anticipated". According to Tepper-García 28] the $y$ values span the range of $10^{-8}$ to $10^{-3}$ for quasar absorption lines, whereas Lynas-Gray [29] expects $y \leq 1$ for stellar absorption lines and considered $10^{-7}<y<10^{3}$.

In Fig. 2 (left) the Voigt function approximations are compared to a reference given by the Weideman [5] rational approximation (" $a$-expansion" with 32 terms) for some $y$. Whereas the approximations appear to be fine for large $y$, clear discrepancies show up for small $y \leq 1$ and intermediate $x$. For the two approximations based on (4) deviations are evident at the origin even for $y=1$. This is clearly confirmed in the plot of the relative deviations $\Delta K(x, y) / K_{\text {ref }}(x, y) \equiv\left|K_{\text {approx }}-K_{\text {ref }}\right| / K_{\text {ref }}$ shown in the right column. Near the origin, the Jiménez-Mier approximations performs slightly better, whereas in the near wings the "Atlas of Functions Voigt" has smaller residuals, and the "correct width" approximations (8) are significantly worse for all $y$. Relative deviations of some $10^{-4}$ for $y=10$ might be acceptable for some applications. However, for large $y$ there are "easier" ways to evaluate the Voigt function: asymptotically the Voigt function approaches the Lorentz function (exploited by several algorithms, e.g. Wells 27, Imai et al. 30]), and for $y>15$ a simple rational approximation with a numerator linear in $x^{2}$ and a denominator polynomial quadratic in $x^{2}$ is better than $10^{-4}$ relative accuracy [3].

The significant accuracy problems of the approximations are also evident in the contour plots of Fig. 3. An accuracy better than $10^{-4}$, considered as appropriate for atmospheric spectroscopy in Schreier [25], is only attained in the Lorentz regime $y>100$. Even for "moderate" values of $y$ the relative 


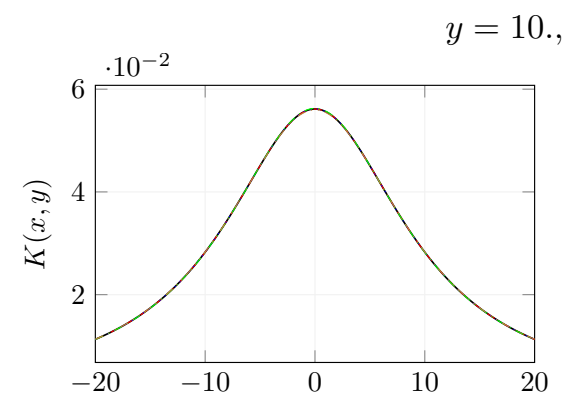

$x_{1 / 2}=10.1$
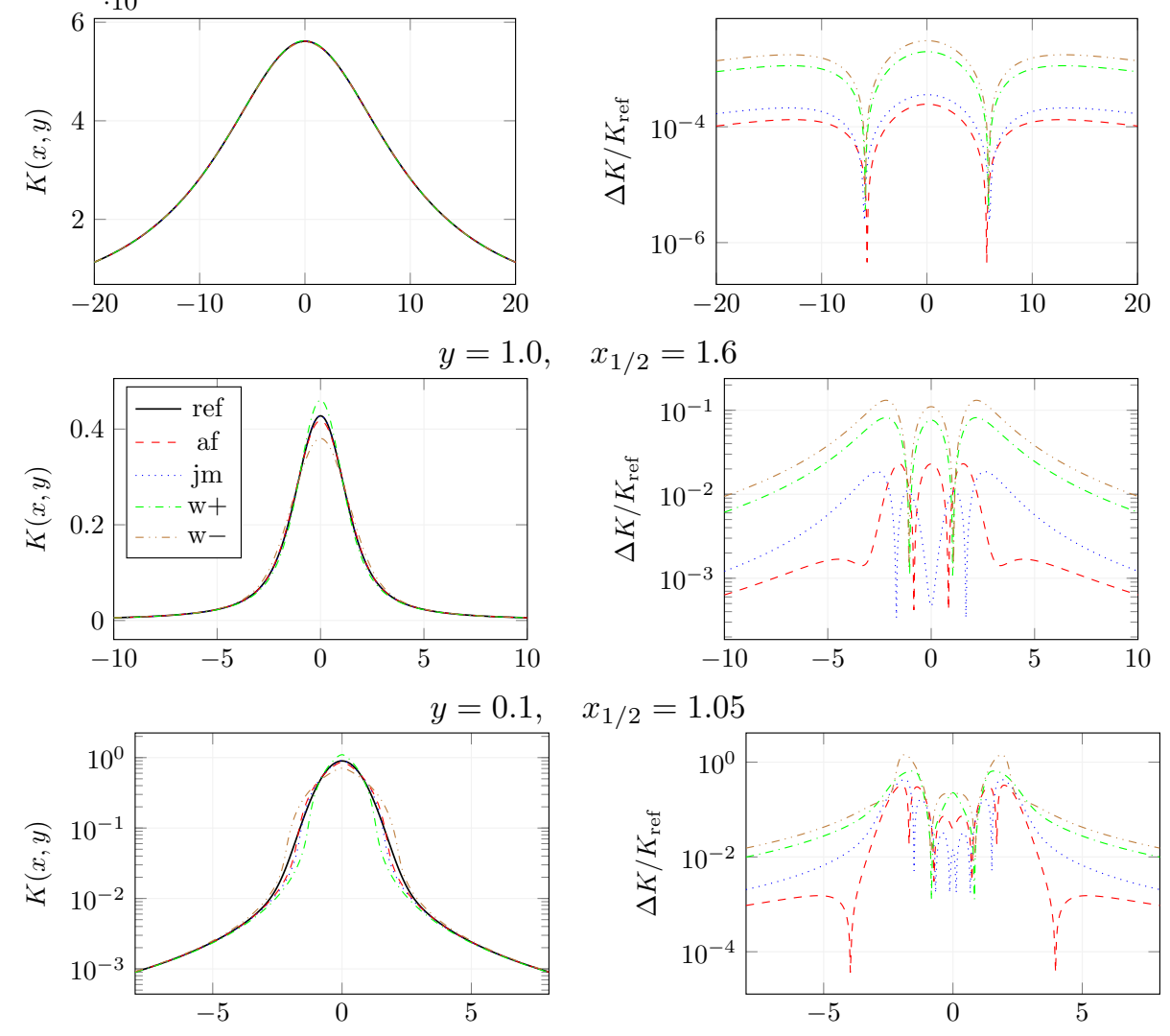

$x_{1 / 2}=1.05$
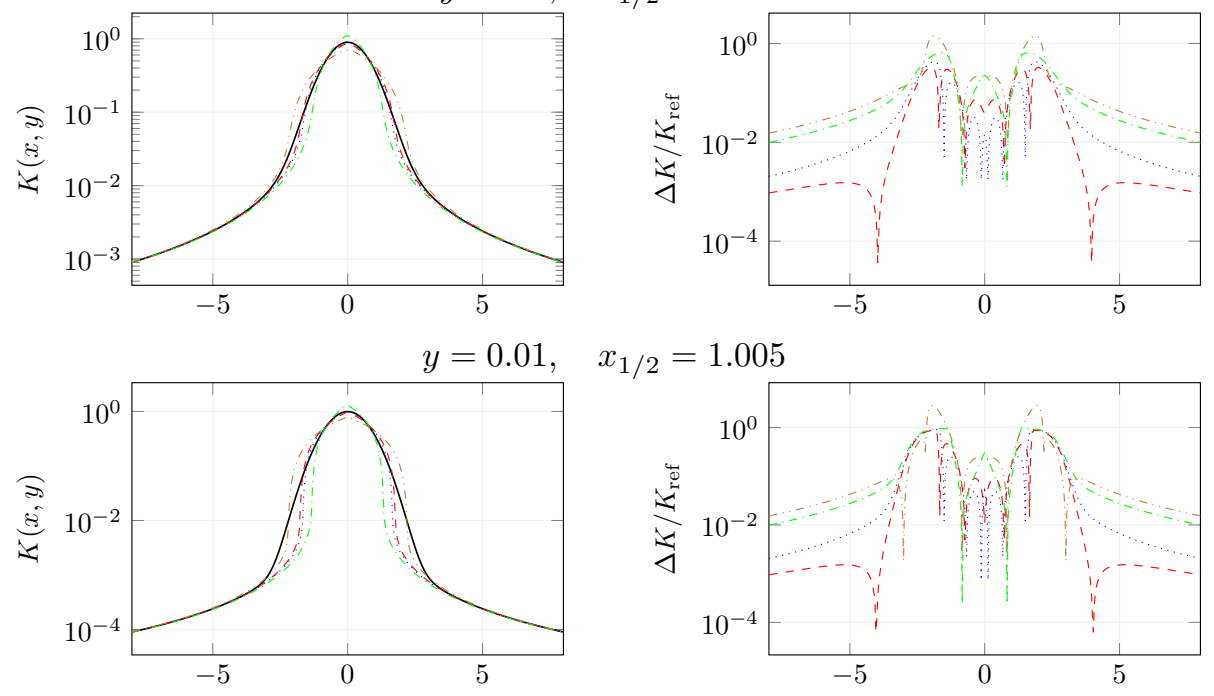

Figure 2: Comparisons for $y=10$ (top), $y=1, y=0.1$, and $y=0.01$ (bottom). Note the different ranges of the $x$ axis. Furthermore a linear $K$ axis is used for the large $y$ cases, whereas a logarithmic axis is used for small $y$. 

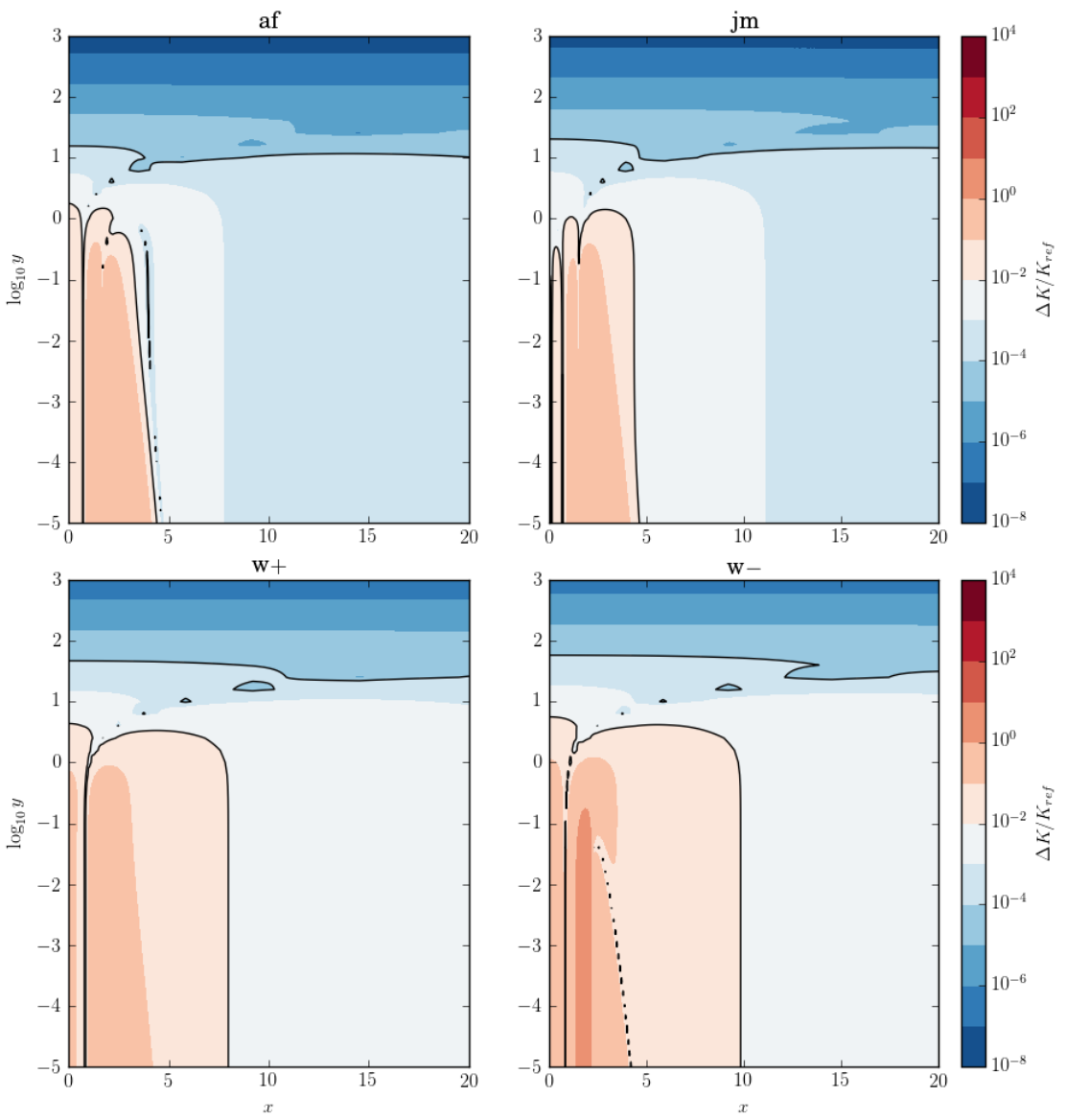

Figure 3: Contour plot of relative differences of the "Atlas of Functions" Voigt approximation (top left), the Jimenez-Mier approximation (top right) and the "correct width" approximations (bottom). The black lines indicate the $10^{-4}$ and $10^{-2}$ error levels. Reddish colors indicate discrepancies larger than $10^{-2}$. 

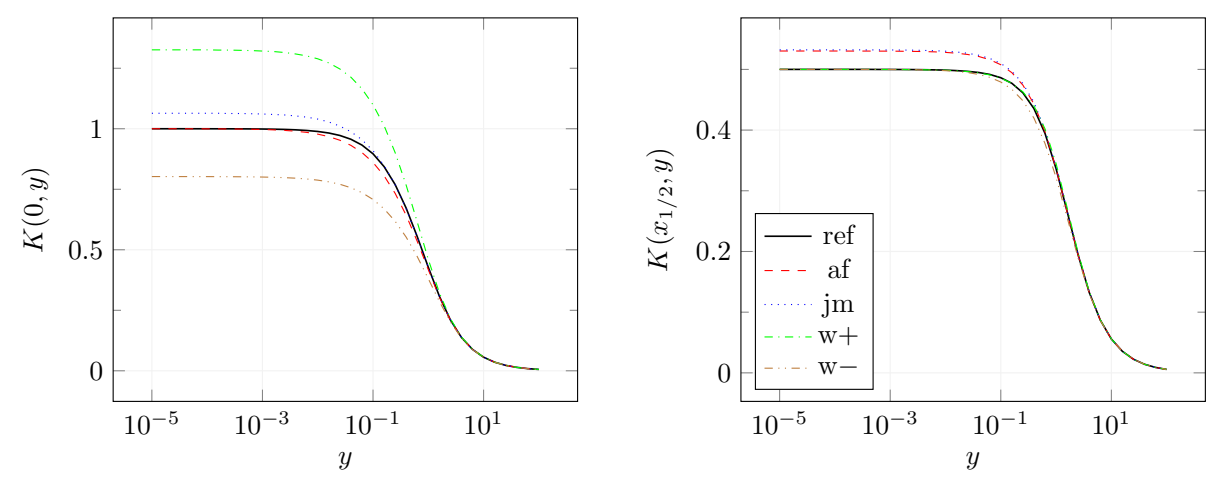

Figure 4: Comparisons for Voigt function values for $x=0$ (left) and $x=x_{1 / 2}=\sqrt{\ln 2}$ (right).

deviations are in the percent range or worse. And for $y \ll 1$ and small $|x|$ deviations are clearly unacceptable.

The problems of the approximations for small $y$-values are further confirmed in Fig. 4. The overestimation of the center value $(x=0)$ of the Jiménez-Mier triangular $(2)$ is translated into an over-estimation of $K(0, y)$ for small $y \ll 1$, whereas the "Atlas of Functions" Voigt appears to be correct except for $y$ in the order of $10^{-1}$. For the approximation (8) the deviations of the center value are even larger and tend to $a_{ \pm}$for $y \rightarrow 0$. On the other hand, for the Voigt function at the half width distance from the line center $\left(x_{1 / 2} \approx\left(y+\sqrt{y^{2}+4}\right) / 2\right.$, [8]) both approximations tend to the correct value $K\left(x_{1 / 2}, y\right)=0.5$ for $y \rightarrow 0$, whereas the Jiménez-Mier and "Atlas of Functions" approximations obviously deviate from the correct value.

For large values of $y$ the definite integrals of the approximations are very close to $\sqrt{\pi}$, but for $y<10^{-2}$ small discrepancies of about $10^{-4}$ compared to the integral of the Weideman-Voigt can be observed.

In view of the six function calls required for each $x, y$ (four calls if the logarithms are combined), these approximations are also likely to be less efficient (Exploiting the arctan addition theorems is tempting, but would require some care). In fact, evaluation of molecular cross sections for some dozen levels in a US Standard atmosphere were about a factor 4 to 5 slower with the "triangular Voigt approximation" compared to the Humliček-Weideman combination of rational approximations [25].

\section{Conclusions}

Several closed-form representations of the Voigt function based on triangular approximations of the Gauss functions have been studied. In contrast to the statement given by Jiménez-Mier 23. this kind of approximation fails to give a useful approach to evaluate the Voigt function accurately except for very large values of the Lorentz to Gauss width ratio $y$. Our timing benchmarks also 
indicated that these approximations are not competitive from a computational efficiency point.

The objective of this note has not been a comprehensive assessment of all Voigt function approximations based on closed-form expressions in terms of elementary functions. These kinds of approximations might be useful in certain applications. Moreover, Klim [31] demonstrated that some approximations, e.g. by Kielkopf [10, can be relatively accurate even for small $y$. Nevertheless, our experience 25] indicates that there are numerous accurate approximations suitable for a wide range of applications. In particular, rational approximations such as Humliček [3] and Weideman [5] are easy to implement and can be very efficient.

\section{References}

[1] Woldemar Voigt. Über das Gesetz der Intensitätsverteilung innerhalb der Linien eines Gasspektrums. In Sitzungsberichte der mathematisch-physikalischen Klasse der Kgl. Bayr. Akademie der Wissenschaften, number 42, pages 603-620, 1912.

[2] B.H. Armstrong. Spectrum line profiles: The Voigt function. J. Quant. Spectrosc. E Radiat. Transfer, 7:61-88, 1967. doi: 10.1016/0022-4073(67)90057-X.

[3] J. Humliček. Optimized computation of the Voigt and complex probability function. J. Quant. Spectrosc. \& Radiat. Transfer, 27:437-444, 1982 . doi: 10.1016/0022-4073(82)90078-4.

[4] G.P.M. Poppe and C.M.J. Wijers. More efficient computation of the complex error function. ACM Trans. Math. Soft., 16:38-46, 1990. doi: 10.1145/77626.77629.

[5] J.A.C. Weideman. Computation of the complex error function. SIAM J. Num. Anal., 31:1497-1518, 1994. doi: 10.1137/0731077.

[6] F.W.J. Olver, D.W. Lozier, R.F. Boisvert, and C.W. Clark, editors. NIST Handbook of Mathematical Functions. Cambridge University Press. Print companion to 7 .

[7] DLMF. NIST Digital Library of Mathematical Functions. National Institute of Standards and Technology. URL http://dlmf.nist.gov/ Online companion to [6].

[8] E.E. Whiting. An empirical approximation to the Voigt profile. J. Quant. Spectrosc. E Radiat. Transfer, 8:1379, 1968. doi: 10.1016/0022-4073(68)90081-2.

[9] J.J. Peyre and G. Principi. Linear combination of Lorentzian and Gaussian profiles to fit resonance spectra. Nucl. Instr. and Meth., 101(3):605-606, 1972. doi: 10.1016/0029-554X(72)90054-7.

[10] J.F. Kielkopf. New approximation to the Voigt function with applications to spectral-line profile analysis. J. Opt. Soc. Am., 63:987-995, 1973. doi: 10.1364/ JOSA.63.000987.

[11] G. K. Wertheim, M. A. Butler, K. W. West, and D. N. E. Buchanan. Determination of the Gaussian and Lorentzian content of experimental line shapes. Review of Scientific Instruments, 45(11):1369-1371, 1974. doi: 10.1063/1.1686503.

[12] P. Thompson, D. E. Cox, and J. B. Hastings. Rietveld refinement of DebyeScherrer synchrotron X-ray data from $\mathrm{Al}_{2} \mathrm{O}_{3}$. J. of Applied Crystallography, 20 (2):79-83, 1987. doi: 10.1107/S0021889887087090. 
[13] C.M. Teodorescu, J.M. Estava, R.C. Karnatak, and A. El Afif. An approximation of the Voigt I profile for the fitting of experimental X-ray absorption data. J. Nucl. Instr. and Meth., A 345:141-147, 1994.

[14] D.V. Titov and R. Haus. A fast and accurate method of calculation of gaseous transmission functions in planetary atmospheres. Planet. Space Sci., 45:369-377, 1997. doi: 10.1016/S0032-0633(96)00129-8.

[15] T. Ida, M. Ando, and H. Toraya. Extended pseudo-Voigt function for approximating the Voigt profile. Journal of Applied Crystallography, 33(6):1311-1316, 2000. doi: 10.1107/S0021889800010219.

[16] Yuyan Liu, Jieli Lin, Guangming Huang, Yuanqing Guo, and Chuanxi Duan. Simple empirical analytical approximation to the Voigt profile. J. Opt. Soc. Am.B, 18(5):666-672, 2001. doi: 10.1364/JOSAB.18.000666.

[17] H. Flores-Llamas, A. Cabrol-Prieto, H. Jiménez-Domínguez, and M. TorresValderrama. An expression for an approximation of the Voigt profile I. Nucl. Instr. and Meth., A 300:159-163, 1991.

[18] H. Melcher and E. Gerth. Darstellung von Linienprofilen durch LorentzFunktionen $n$-ten Grades. Experimentelle Technik der Physik, 25:527-538, 1977.

[19] P. Martin and J. Puerta. Generalized Lorentzian approximations for the Voigt line shape. Appl. Opt., 20:259-263, 1981. doi: 10.1364/AO.20.000259.

[20] P. Martin and J. Puerta. Generalized Lorentzian approximations for the Voigt line shape: errata. Appl. Opt., 20:2601, 1981. doi: 10.1364/AO.20.002601.

[21] J. Puerta and P. Martin. Three and four generalized Lorentzian approximations for the Voigt line shape. Appl. Opt., 20:3923-3928, 1981. doi: 10.1364/AO.20. 003923.

[22] J. Puerta and P. Martin. Three and four generalized Lorentzian approximations for the Voigt line shape: errata. Appl. Opt., 22:19, 1983. doi: 10.1364/AO.22. 000019 .

[23] J. Jiménez-Mier. An approximation to the plasma dispersion function. J. Quant. Spectrosc. ES Radiat. Transfer, 70(3):273-284, 2001. doi: 10.1016/S0022-4073(00) 00139-4.

[24] Keith B. Oldham, Jan Myland, and Jerome Spanier. An Atlas of Functions. Springer, 2009. doi: 10.1007/978-0-387-48807-3.

[25] F. Schreier. Optimized implementations of rational approximations for the Voigt and complex error function. J. Quant. Spectrosc. \& R Radiat. Transfer, 112(6): 1010-1025, 2011. doi: 10.1016/j.jqsrt.2010.12.010.

[26] Kendra L. Letchworth and D. Chris Benner. Rapid and accurate calculation of the Voigt function. J. Quant. Spectrosc. \& Radiat. Transfer, 107:173-192, 2007. doi: $10.1016 /$ j.jqsrt.2007.01.052.

[27] R.J. Wells. Rapid approximation to the Voigt/Faddeeva function and its derivatives. J. Quant. Spectrosc. \& Radiat. Transfer, 62:29-48, 1999 . doi: 10.1016/S0022-4073(97)00231-8.

[28] Thorsten Tepper-García. Voigt profile fitting to quasar absorption lines: An analytic approximation to the Voigt-Hjerting function. Mon. Not. Roy. Astron. Soc., 369:2025-2035, 2006. doi: 10.1111/j.1365-2966.2006.10450.x.

[29] A.E. Lynas-Gray. VOIGTL - a fast subroutine for Voigt function evaluation on vector processors. Comp. Phys. Comm., 75:135-142, 1993. doi: 10.1016/ 
0010-4655(93)90171-8.

[30] K. Imai, M. Suzuki, and C. Takahashi. Evaluation of Voigt algorithms for the ISS/JEM/ SMILES L2 data processing system. Adv. Space Res., 45:669-675, 2010. doi: 10.1016/j.asr.2009.11.005.

[31] A. Klim. A comparison of methods for the calculation of Voigt profiles. J. Quant. Spectrosc. \& Radiat. Transfer, 26:537-545, 1981. doi: 10.1016/0022-4073(81) 90041-8. 